\title{
Free at last!
}

\section{Dennis P. Curran invites everyone to join the dawning new era of organic synthesis.}

Organic synthesis has always suffered from many constraints, and it is often stated that we are still a long way from routine 'ideal reactions'. But have you noticed yet the liberating groundswell that is now occurring? Researchers have recently discovered acid-free reactions and base-free reactions. And there are reactions that are metal-free, catalyst-free, protecting-group-free, solvent-free, and so on.

Professor Armido Studer and I recently complained that some transition-metal-free reactions looked eerily like well-established radical homolytic substitutions and $\mathrm{S}_{\mathrm{RN}} 1$ reactions ${ }^{1}$. But since then, I have come to see that this kind of small-minded thinking is hindering the dawn of a new era of organic synthesis. In this brave new world, every reaction may not yet be an ideal reaction, but at least any paper can be an ideal paper.

Take an example from my own group's research. We have recently obtained astonishing preliminary results showing that entirely transition-metal-free hydrolysis of esters can be accomplished with only inexpensive sodium hydroxide and the 'greenest' solvent of all - water! You won't be surprised to learn that we had to overcome major obstacles to make a discovery of this magnitude. To give just one example, it was an almost insurmountable challenge to find the water required for the reaction. (It is often said that water is a naturally green solvent, but most of the naturally occurring water in the Pittsburgh area is brown.)

Prior to publication, we just have to prove that our samples of sodium hydroxide and

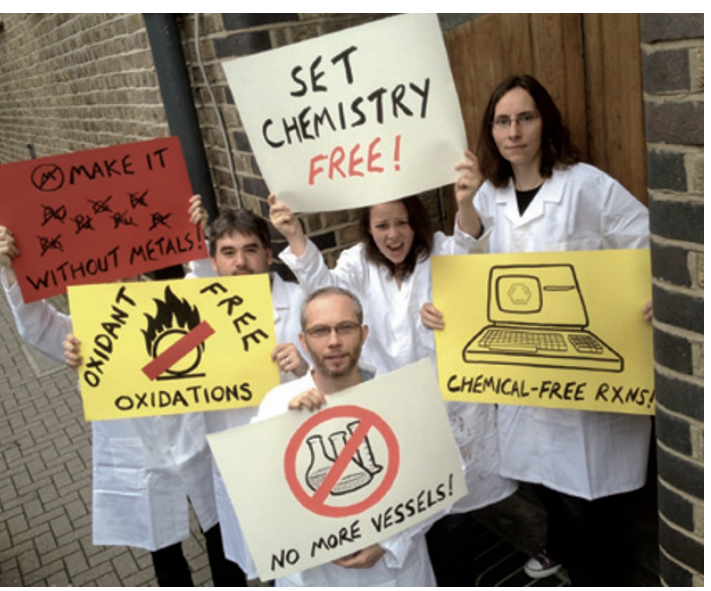

Cutting-edge researchers forcefully espousing a new vision of organic synthesis. water are not contaminated with traces of transition metals. This remarkable discovery will surely qualify for publication in Nature Chemistry as long as the reviewers and editors consider the work in its proper context (the topical area of transition-metal-free reactions). And this is only the start. Clearly the freedom wave is too big for one group, so I will share just a few of our ideas. We are unafraid of competition; on the contrary, I invite you to join this hot and burgeoning field.

\section{In this brave new world, every reaction may not yet be an ideal reaction, but at least any paper can be an ideal paper.}

First, following the precedent of solventfree reactions, we will develop vesselfree, pressure-free and temperature-free reactions. Vessel-free reactions are not so important for academic research, but just imagine what will happen in industry when the inevitable spillover occurs. Pressure-free reactions will have explosive impact, and the energy savings from temperature-free reactions are literally inestimable.

Then, with the precedent of catalystfree reactions, we will go for the really big goals, reagent-free, reactant-free and substrate-free reactions. Imagine such titles as 'Oxidant-free oxidations', 'Diene- and dienophile-free Diels-Alder reactions', and so on. Undoubtedly a gold-free gold mine of Science and Nature papers! A naysaying reviewer of my recent grant proposal suggested that such reactions would also be product-free. This is research of course, so we cannot predict the results in advance. But even if true on rare occasions, this small detraction will be more than offset because such reactions are waste-free and cost-free.

This refreshing wave of liberation will extend beyond reactions proper. For example, organofluorine chemists have recently complained that they have to cite prior work in fluorine chemistry, but that newcomers who dabble in the field do not ${ }^{2}$. To address this patently unfair situation, we are starting the 'Society of Fluorine-Free Chemistry'. Any member of this society, regardless of background and training, will be allowed to do research and publish in fluorine chemistry without saying anything at all about prior art in the field. The freedom to do this will greatly expedite publication of new research, up to and including the discovery of the long elusive elemental fluorine (an achievement that could surely land a Nobel Prize).

Named reactions are also subject to study. Examples include the Huisgen-free 1,3-dipolar cycloaddition reaction (which may already be known) and the Kishi-free NHK reaction (alternatively called the NozakiHiyama reaction). Some inappropriate 'free' names might also need to be replaced. For example, we propose that 'click-free' should instead be 'clack'. The broader field then becomes 'click and clack reactions'. In rare cases, the term 'anti' is preferred over 'free', as in the 'anti-NOC' (nitrile oxide cycloaddition) reaction; obviously this reaction cannot be conducted under lead-free conditions. You might know that the freedom wave has already struck other areas of science and technology. For example, for those of you reading on a screen, this is a paper-free paper.

By now you will have guessed that the nec plus ultra in the new era is reactionfree reactions. Computational chemists will grouse that they have been doing such things in silico for decades. But this is missing my whole point. What I'm saying is that for far too long now, authors of papers in organic synthesis have been telling their readers what their work is about and what is in their reactions. As more and more has become known, this approach has become too constraining. Outdated, in a word.

In the future, authors will be free to tell readers what their work is not about and what is not in their reactions. Our new transition-metal-free ester hydrolysis reaction is not a rechristening, but a veritable rebirth. This is nothing less than the dawn of a new utopia for all authors the era of the precedent-free publication.
Dennis P. Curran is in the Department of Chemistry at the University of Pittsburgh, Pittsburgh, Pennsylvania 15260, USA. He is the author of over 400 other humour- free articles, many appearing in JACS-free the important area of tin-free chemistry. e-mail:curran@pitt.edu

\footnotetext{
References

1. Studer, A. \& Curran, D. P. Angew. Chem. Int. Ed 50, 5018-5022 (2011).

Ritter, S. K. Chem. Eng. News 90, 10-17 (2012).
} journals. Most of his research now focuses on 